PACS: 78.67.-n, 78.30-j, 78.55.-m.

\title{
The nature of red emission in porous silicon
}

\author{
L.Yu. Khomenkova, N.E. Korsunska, B.M. Bulakh, M.K. Sheinkman, T.R. Stara \\ V. Lashkaryov Institute of Semiconductor Physics, NAS of Ukraine \\ 41, prospect Nauky, 03028 Kyiv, Ukraine \\ Phone: +38 04452572 34; Fax: +38 0445258342 \\ E-mail: khomen@ukr.net
}

\begin{abstract}
The photoluminescence spectra of porous silicon at 77 and $300 \mathrm{~K}$ and their transformation during aging were investigated. The competition of two radiative recombination channels that have a common excitation mechanism was observed. It is shown that only one of them, which causes infrared emission band and is present in asprepared samples, is connected with excitonic recombination in $\mathrm{Si}$ nanocrystals. The second one that causes red emission band appears during aging is supposed to be connected with carrier recombination through oxide-related defects. It is shown that this channel dominates in aged samples.
\end{abstract}

Keywords: porous silicon, photoluminescence, recombination.

Manuscript received 01.12.04; accepted for publication 18.05.05.

\section{Introduction}

One of the most important theoretical and experimental tasks in physics of silicon-based nano-structures is the clarification of the nature of radiative transitions. One of such systems is well-known porous silicon (PSi) that shows bright red emission at room temperature. In spite of numerous investigations, the nature of this emission is currently under discussion [1 - 13]. One of the most widespread models of red emission is excitonic recombination in quantum confined silicon nanocrystals [9]. However, a number of experimental results are hardto-explain within this model. For example, the red band (R-band) demonstrates a different behavior in dependence on preparation conditions and further treatments. In particular, under cooling the peak position of red photoluminescence (PL) shifts to the high-energy side in different samples, to the low-energy one [8] or doesn't change [5]. Such a behavior of this band is usually explained by the contribution of different radiative channels to PL spectrum.

Beside, the excitonic recombination inside $\mathrm{Si}$ nanocrystals, another radiative channels such as electron-hole recombination through oxide-related centers [5 - 7] or surface states [10] as well as recombination of the excitons localized at $\mathrm{Si}=\mathrm{O}$ bonds [11] or in quantum confinement well at $\mathrm{Si} / \mathrm{SiO}_{2}$ interface [8] were considered.

However, it is impossible to consider any of the above-mentioned models to be proved completely, since the PL components resulted from different recombination channels were not observed simultaneously as separated bands and their possible contribution to wide emission spectrum observed in porous Si was not extracted.

Different approaches can be used to discriminate the radiative channels. One of them is the study of PL spectra dependence on Si crystallite sizes when the latter vary in a wide range. We used such an approach for investigation of the emission properties of $\mathrm{Si}-\mathrm{SiO}_{2}$ layers prepared by magnetron sputtering $[12,13]$. It was shown that, at room temperature, the PL spectra of these layers consist of several overlapping bands. The shift of peak position with the change of $\mathrm{Si}$ nanocrystal sizes was found only for the infrared PL band, while the peak positions of another ones were constant. The latter bands were attributed to silicon oxide defects. However, for porous silicon, PSi, such an approach is low-effective because PL spectra at room temperature are broad and unstructured.

In this work, another approach based on the investigation of the temperature behavior of PL spectra and their transformation during PSi aging was used to clarify the contribution of different radiative channels to red emission.

\section{Experimental procedures}

The PSi layers were prepared by the electrochemical etching of Si substrate (boron-doped, $\rho=10 \mathrm{Ohm} \cdot \mathrm{cm}$, (100) orientation) in non-aqueous HF-ethanol electrolyte $\left(\mathrm{HF}: \mathrm{C}_{2} \mathrm{H}_{5} \mathrm{OH}=2: 1\right)$ at the current density $j_{\mathrm{A}}=50 \mathrm{~mA} / \mathrm{cm}^{2}$ and time duration $t_{\mathrm{A}}=10 \mathrm{~min}$. PL and PL excitation (PLE) spectra were measured at 77 and $300 \mathrm{~K}$ with the setup described in $[5,12,14]$ under excitation by a xenon lamp. To control Si nanocrystal 

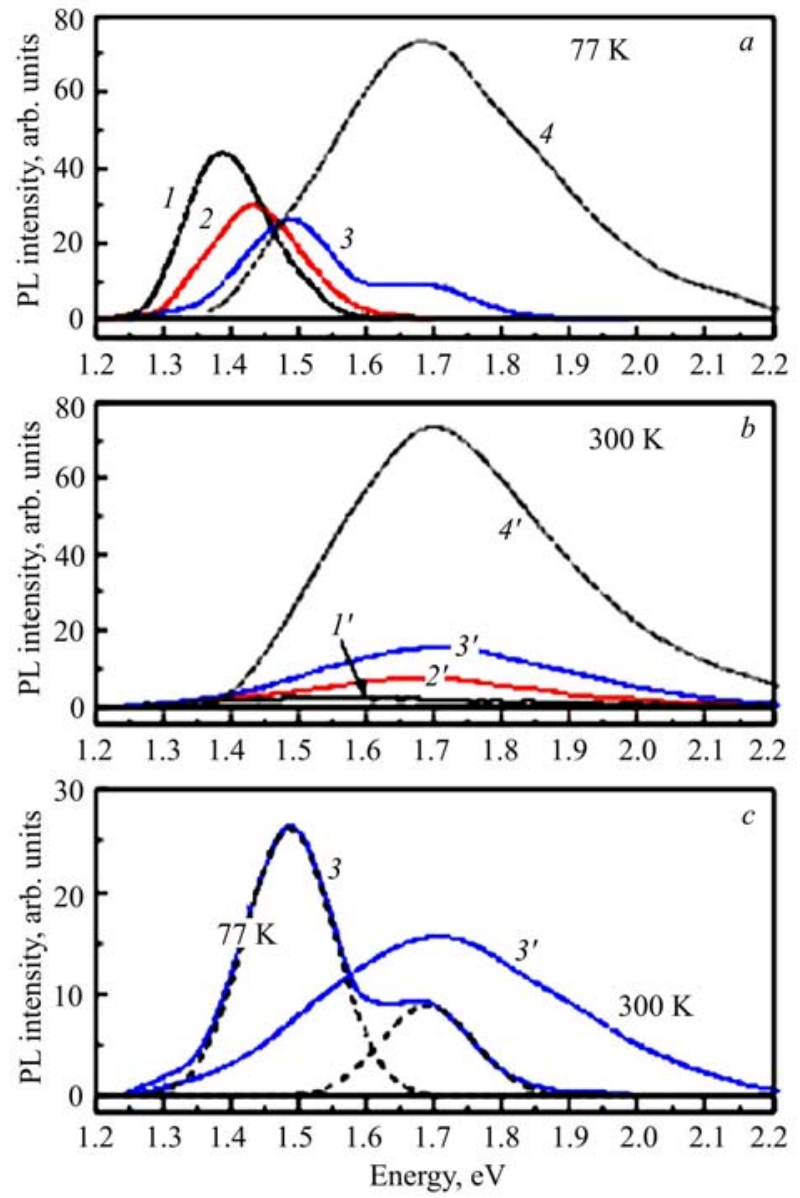

Fig. 1. PL spectra of PSi layer: as-prepared (1) and after aging during 2 hours (2), 15 days (3) and 150 days (4) measured at 77 (a) and $300 \mathrm{~K} \mathrm{(b);} \mathrm{c} \mathrm{-} \mathrm{the} \mathrm{comparison} \mathrm{of} \mathrm{curves} 3$ from Figs $1 \mathrm{a}$ and $\mathrm{b}$.

sizes, the Raman scattering spectra were measured when exciting by $487.9 \mathrm{~nm}$ line of the argon laser LGI-503 and detected with the setup based on a DFS-24 spectrometer with a cooled FEU-136 photomultiplier in the photon-counting mode. To avoid the layer heating, the power density of laser emission did not exceed $1.5 \mathrm{~W} / \mathrm{cm}^{2}$. The Raman spectra measurements were carried out at the room temperature.

\section{Experimental results}

The low-temperature PL spectra of PSi layers are shown in Fig. 1a. The as-prepared samples demonstrate one infrared emission band (IR-band) with a full-width at half-maximum (FWHM) $\sim 0.14 \mathrm{eV}$ (Fig. 1a, curve 1). The aging in air for up to two weeks leads to the decrease of PL intensity and shift of its peak to the highenergy side (curves 2, 3). Simultaneously, the tightening of high-energy PL edge to the high-energy side and then the appearance of an additional PL band (R-band) is observed. As follows from decomposition of curve 3

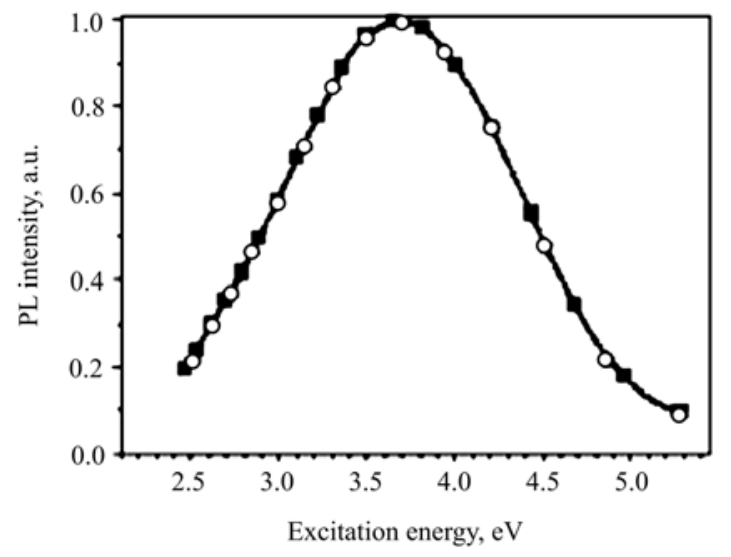

Fig. 2. Normalized PLE spectra of 15-days aged sample measured at $300 \mathrm{~K}$ for $1.4 \mathrm{eV}$ (squares) and $1.8 \mathrm{eV}$ (circles). The excitation energy is $3.5 \mathrm{eV}$.

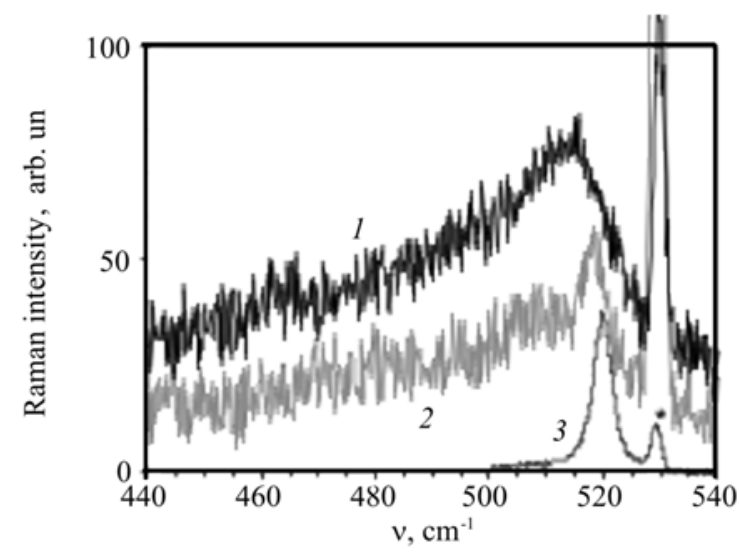

Fig. 3. Raman scattering spectra of as-prepared (1) and 15days aged (2) samples. The curve 3 corresponds to the Raman scattering from Si substrate.

from Fig. 1a (Fig. 1c), this R-band peaks at $\sim 1.69 \mathrm{eV}$ and has the FWHM $\sim 0.2 \mathrm{eV}$. After more prolong aging, the PL intensity increases essentially, and again its spectrum contains only one band, the maximum of which shifts to the high-energy side up to $1.7 \mathrm{eV}$ after 150 days aging (curves 4, 5). However, its FWHM $(0.35 \ldots 0.4 \mathrm{eV})$ essentially exceeds the FWHM of the PL band in the as-prepared sample.

At $300 \mathrm{~K}$, the PL intensity in as-prepared samples is very low, and the PL spectra contain one infrared band (Fig. 1b, curve 1). Aging for 2 hours leads to the increase of the PL intensity and to shift of the PL band peak position from 1.55 to $1.7 \mathrm{eV}$. After more prolong aging, the PL intensity increases, while the peak position remains constant. PLE spectra of both bands coincide (Fig. 2, curve 1) and show one broad band peaking at $3.5 \mathrm{eV}$.

The Raman scattering spectra of as-prepared samples show a broad band with the peak at $513 \mathrm{~cm}^{-1}$ (Fig. 3, curve 1). After 15-days aging, the Raman band shifts to 
low-frequency side down to $508 \mathrm{~cm}^{-1}$. Besides, the additional band at $519 \mathrm{~cm}^{-1}$ appears (curve 2). Obviously, this band results from substrate (Fig. 3, curve 3), and its appearance is due to the increase of layer transparency during oxidation. The estimation of $\mathrm{Si}$ nanocrystal sizes with using the calculation [15] shows that, in as-prepared samples, the sizes of Si nanocrystals are $4 \mathrm{~nm}$ while in the 15-days aged samples they are only $3 \mathrm{~nm}$.

\section{Discussion}

The above-mentioned results show that the high-energy shift of the IR-band peak position correlates with the decrease of Si nanocrystal sizes during aging. So, we can ascribe IR-band to excitonic recombination inside $\mathrm{Si}$ nanocrystals.

During aging, an additional R-band appears in the low-temperature PL spectra, and its intensity increases with the aging time (Fig. 1a, curves 2, 3). However, after the prolong aging only one band is observed in the PL spectra again. Because of its high FWHM, we can think that this band is the superposition of IR- and R-ones. It is confirmed by the shift of broad band peak position with the aging time to $1.7 \mathrm{eV}$ that coincides with the peak position of R-band (Fig. 1a, curve 3). The last fact means that R-band dominates in low-temperature PL spectra after 150 days aging, and its peak position is independent of aging time and crystallite sizes. As Fig. $1 \mathrm{~b}$ shows, the R-band dominates at $300 \mathrm{~K}$ after 2 hours aging. The coincidence of the PL peak positions at 77 and $300 \mathrm{~K}$ after prolong aging means that the R-band peak position is temperature independent. In general case, this transformation of PL spectra during aging and with temperature can be explained by the change of IR- and R-bands contribution.

Since the R-band appears under natural oxidation and its maximum position is independent of temperature, it is possible to attribute this band to carrier recombination through oxide-related centers. This is confirmed by the observation of such a band in silicate glasses [10].

The intensity of IR-band decreases at the first stage of aging. At the same time, the decomposition of curve 4 in Fig. 1a (not shown) indicates that its intensity increases after prolong aging. As we have shown earlier [14], the as-prepared samples are highly passivated, while during aging the $P_{b}$-centers appear due to $\mathrm{Si} / \mathrm{SiO}_{2}$ interface formation. So, we can attribute the decrease of IR-band intensity at the beginning of aging to the increase of the number of $P_{b}$-centers that are the centers of non-radiative recombination. At the same time, the increase of the IR-band intensity can be a result of the barrier height increase, and the crystallite sizes decrease due to the crystallite oxidation [17].

When IR- and R-band are observed separately (1 15 days aged samples), we can see that these bands have the reverse temperature dependences: when heating the IR-band intensity quenches while the R-band one enhances (Fig. 1c). As the PLE spectra of these bands are similar (Fig. 2), we can conclude that they have a common excitation mechanism that is obviously the light absorption in $\mathrm{Si}$ nanocrystals $[7,12,16]$. In this case, the reverse temperature dependence of IR- and Rband intensities can be caused by a competition of recombination flows through $\mathrm{Si}$ nanocrystals and oxiderelated centers.

\section{Conclusions}

It is shown that the competition of two radiative channels takes place in PSi samples. One of them that causes the infrared band is due to the excitonic recombination in Si nanocrystals. Another one can be attributed to carrier recombination through oxide-related defects and causes the red emission. It is shown that both bands are excited due to light absorption by $\mathrm{Si}$ nanocrystals, and the competition of radiative recombination in $\mathrm{Si}$ nanocrystals as well as in oxiderelated defects takes place.

\section{References}

1. D.I. Kovalev, I.D. Yarostietzkii, T. Muschik, Fast and slow visible luminescence bands of oxidized porous Si // Appl. Phys. Lett. 64 (2), p. 214-216 (1994).

2. L. Tsybeskov, Yu.V. Vandyshev, P.M. Fauchet, Blue emission in porous silicon: Oxygen-related photoluminescence // Phys. Rev. B 49 (11), p. 7821 7824 (1994).

3. H.D. Fuchs, M. Stutzmann, M.S. Brandt, M. Rosenbauer, J. Weber, A. Breischwerdt, P. Deak, M. Cardona, Porous silicon and siloxene: Vibrational and structural properties // Ibid. 48 (11), p. 8172-8189 (1993).

4. G.G. Qin, Y.Q. Jia, Mechanism of visible luminescence in porous silicon // Solid State Communs $\mathbf{8 6}$ (9), p. 559-563 (1993).

5. S.M. Prokes, Light emission in thermally oxidized porous silicon: Evidence for oxide-related luminescence // Appl. Phys. Lett. 62 (25), p. 3244-3246 (1993).

6. T.V. Torchynska, M. Morales Rodriguez, F.G. Bacarril-Espinoza, N.E. Korsunskaya, L.Yu. Khomenkova, L.V. Scherbina, Ballistic effect in red photoluminescence of Si wires // Phys. Rev. B 65, p. 115313-1 - 115313-7 (2002).

7. N. Korsunska, M. Baran, L. Khomenkova, V. Yukhymchuk, Y. Goldstein, E. Savir, J. Jedrzejewski, Mechanism of photoexcitation of oxide-related emission bands in $\mathrm{Si}_{-} \mathrm{SiO}_{2}$ systems // Mater. Sci. and Eng. C 23, p. 691-696 (2003).

8. Y. Kanemitsu, S. Okamoto, Resonantly excited photoluminescence from porous silicon: Effects of 
surface oxidation on resonant luminescence spectra // Phys. Rev. B 56 (4), p. R1696 - R1699 (1997).

9. G. Cullis, L.T. Canham, P.D.J. Calcott, The structural and luminescence properties of porous silicon // J. Appl. Phys. 82 (3), p. 909-965 (1997).

10. F. Koch, V. Petrova-Koch, T. Muschik, The luminescence of porous $\mathrm{Si}$ : the case for the surface state mechanism // J. Lumin. 57, p. 271-282 (1993).

11. Murayama, S. Miyazaki, M. Hirose, Excitation and recombination processes in porous silicon // Solid State Communs 93, p. 841-846 (1995).

12. N. Korsunska, L. Khomenkova, V. Yukhimchuk, B. Jumayev, T. Torchynska, A. Many, E. Savir, Y. Goldstein, J. Jedrzejewski, Defect-related luminescence of $\mathrm{Si} / \mathrm{SiO}_{2}$ layers // J. Phys.: Condens. Mater. 14, p. 13217-13221 (2002).

13. T.V. Torchynska, F.G. Bacarril-Espinoza, Y. Goldstein, E. Savir, J. Jedrzejewskii, L.Yu. Khomenkova, N. Korsunska, V. Yukhimchuk, Nature of visible luminescence of co-sputtered $\mathrm{Si}_{-} \mathrm{SiO}_{\mathrm{x}}$ systems // Physica B 340-342, p. 1119-1123 (2003).
14. Korsunska, M. Baran, B. Bulakh, L. Khomenkova, V. Yukhimchuk, V. Papusha, Influence of $\mathrm{Si} / \mathrm{SiO}_{x}$ interface formation on luminescence characteristics of $\mathrm{Si}_{-} \mathrm{SiO}_{2} / /$ Izv. RAN, Ser. Fiz. 67 (2), p. 223-227 (2003).

15. I.H. Campbell, P.M. Fauchet, The effect of microcrystal size and shape on one phonon raman spectra of crystalline semiconductors // Solid State Communs 58 (10), p. 739-741 (1986).

16. N. Korsunska, L. Khomenkova, V. Yukhimchuk, B. Jumayev, T. Torchynska, A. Many, E. Savir, Y. Goldstein, J. Jedrzejewski, Nature of visible luminescence and its excitation in $\mathrm{Si}_{-} \mathrm{SiO}_{\mathrm{x}}$ systems // J. Lumin. 102-103, p. 705-711 (2003).

17. Yu.V. Kryuchenko, and A.V. Sachenko, Quantum efficiency of exciton luminescence in lowdimensional structures with the indirect energy gap // Physika E 14, p. 299-312 (2002). 